\title{
Viscous and inviscid matching of three-dimensional free-surface flows utilizing shell functions
}

\author{
J. Andrew Hamilton • Ronald W. Yeung
}

Received: 30 April 2010 / Accepted: 8 November 2010 / Published online: 18 January 2011

(C) The Author(s) 2011. This article is published with open access at Springerlink.com

\begin{abstract}
A methodology is presented for matching a solution to a three-dimensional free-surface viscous flow in an interior region to an inviscid free-surface flow in an outer region. The outer solution is solved in a general manner in terms of integrals in time and space of a time-dependent free-surface Green function. A cylindrical matching geometry and orthogonal basis functions are exploited to reduce the number of integrals required to characterize the general solution and to eliminate computational difficulties in evaluating singular and highly oscillatory integrals associated with the free-surface Green-function kernel. The resulting outer flow is matched to a solution of the Navier-Stokes equations in the interior region and the matching interface is demonstrated to be transparent to both incoming and outgoing free-surface waves.
\end{abstract}

Keywords Integral equations · Open-boundary condition · Pseudo-spectral solutions ·

Time-dependent free-surface Green function · Viscous-inviscid matching · Wave-body interaction

\section{Introduction}

Including the effects of viscosity in the numerical analysis of wave-body interaction problems involves several challenges, including the large computational domain that is required to include a fast-moving wave-field. An attractive remedy is to limit the viscous solution to the region near the body where viscous effects are most important and to rely on inviscid solutions in an outer region to account for wave behaviors. In addition to the proper procedure to model the matching interface, this approach requires an efficient means to solve for the outer-flow solution which can become time-consuming, even for inviscid-flow solutions if not treated carefully.

Dedication: In fond memory of my friend Ernie who kindly invited me to visit Adelaide in 1981 and provided the environment for the completion of my "Annual Review” article.

R. W. Yeung.

\section{J. A. Hamilton}

Research and Development Division, Monterey Bay Aquarium Research Institute, Moss Landing, CA 95039, USA

e-mail: hamilton@mbari.org

R. W. Yeung $(\varangle)$

Department of Mechanical Engineering, University of California at Berkeley, Berkeley, CA 94720-1740, USA

e-mail: rwyeung@berkeley.edu 
Because the matching boundary between the inner and outer regions can be selected at will and does not depend on body geometry, opportunities exist to compute a generalized solution to the outer flow surface that can be computed once and applied to a wide variety of interior flows. This paper provides details of a particularly efficient technique to formulate this exterior flow problem so that only a minimum collection of integrals of a free-surface Green function are required to characterize all possible outer flows. This technique relies on decomposing the exterior flow characteristics in terms of orthogonal basis functions on a cylindrical matching surface. The particular decomposition chosen provides two distinct advantages; the number of coefficients required to characterize the general outer flow is reduced through the use of Fourier shift identities and, perhaps more importantly, the known geometry and choice of basis functions decreases the singular nature of the Green function and has favorable effects on the evaluation of those functions which is commonly a difficult issue in using Green functions to solve freesurface flows. The third section of the paper describes these techniques in detail after the general solution to the outer flow is outlined in Sect. 2.

With the inviscid outer flow characterized in a general way, the other challenge is matching this flow to a solution of the viscous-flow problem in the interior region. Conceptually this is done by imagining a cylindrical boundary on the interior flow that exerts pressure on the interior flow in a manner that is appropriate to whatever waves would exist in the exterior region. There are several ways to perform this matching and an explicit time-stepping method is described that is consistent with the numerical approach taken to solve the Navier-Stokes equations in the interior region. The technique is demonstrated by solving the flow for a viscous flow around a cylinder in the interior region that is excited by waves propagating inward from the outer solution domain. These waves are shown to pass through the matching boundary, excite the interior flow, diffract around a vertical cylinder, and finally pass back out of the interior region without reflection at the interface.

Previous work on viscous-inviscid domain-decomposition methods have been largely limited to cases without a free-surface boundary $[1,2]$ or cases where the decomposed domains do not intersect the free surface [3]. Works that include a domain decomposition in which the domains intersect the free surface are concentrated on steady flows around a body with forward speed through a water-air interface [4,5]. In contrast, this paper addresses the threedimensional unsteady case of free-surface flows about a body with zero forward speed and builds upon previous work [6].

\section{Generalized solution of outer flow}

The shell method of solution for the outer flow is a general solution to a linearized, inviscid, unsteady flow in the outer region. The complete solution is expressed in terms of coefficients which are integrals in time and space of a Green function. The solution method is specifically developed to represent a general solution of the outer flow which can be later matched to any interior flow, providing an outer boundary condition for the interior flow.

\subsection{Mathematical problem for outer flow}

As illustrated in Fig. 1, the outer fluid domain $V_{o}$ is bounded by a cylindrical matching surface $S_{S}$, the undisturbed free surface $S_{F}$, the bottom $S_{H}$, and a far-field surface $\Sigma$, located at infinity. All quantities are nondimensionalized by a characteristic length $\tilde{L}$, a characteristic velocity $\tilde{U}$, and fluid density $\tilde{\rho}$, where the tilde indicates dimensional variables. For each problem studied, $\tilde{U}$ and $\tilde{L}$ can be chosen as appropriate and will be specified as needed.

The velocity of the fluid is given by the gradient of a velocity potential that satisfies the Laplace equation at all time in the fluid domain and appropriate boundary:

$$
\begin{aligned}
& \nabla^{2} \varphi(P, t)=0, \quad P \in V_{o}, \\
& \varphi_{t t}(P, t)+\varphi_{z}(P, t)=0, \quad P \in S_{F_{o}} .
\end{aligned}
$$




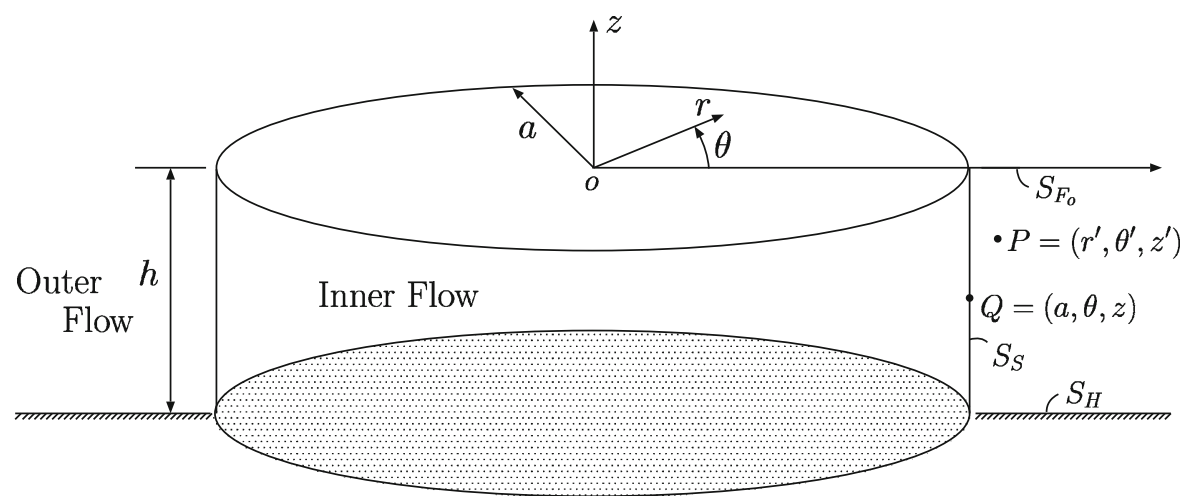

Fig. 1 Schematic of shell geometry

The choice $\tilde{U}=\sqrt{\tilde{g} \tilde{L}}$ has eliminated the usual gravity factor in this equation. Also

$\varphi_{z}(P, t)=0, \quad P \in S_{H}$.

In the far field $\Sigma($ at $\infty)$ the fluid velocities must become vanishingly small and the initial conditions must be supplied.

$\varphi=0, \quad t \leq 0, \quad P \in V_{o}$,

$\eta=-\frac{\partial \varphi}{\partial t}=\eta_{0}, \quad t \leq 0, \quad P \in S_{F}$.

The goal of this outer-problem formulation is to solve the problem defined by (1)-(4) in a general way to provide a relation between $\varphi$ and $\varphi_{r}$ on $S_{S}$ which be used as an outer boundary condition for a variety of interior problems and solution methods.

\subsection{Integral-equation formulation}

The hydrodynamic problem defined above is best approached by using a Green function that satisfies some of the boundary conditions to convert the Laplace equation (1) to an integral equation [7, Sect. 22]. By applying Green's theorem to the time derivative of the potential and the time-dependent Green function, and integrating with respect to time, a time-dependent integral-equation formulation can be obtained [8]. An unsteady Green function $G(P, Q, t-\tau)$ is used which represents the velocity potential at time $t$ and position $Q$ due to a source in the presence of a free surface that comes into existence at time $\tau$ and is located at $P$.

This Green function satisfies the field equation unless $P=Q$, a no-leak condition on the bottom surface, and the linearized free-surface condition:

$\nabla^{2} G(P, Q, t-\tau)=\delta(P-Q), \quad G_{\tau \tau}+\left.G_{z}\right|_{z=0}=0,\left.\quad G_{z}\right|_{z=-h}=0$.

Such a function is given by Wehausen and Laitone [7, p. 604, Eq. 22.3] as the sum of a Rankine part and a time-dependent part:

$G(P, Q, t-\tau)=G^{r}(P, Q)+H(P, Q, t-\tau)$,

where $G^{r}$ is the simple free-space (or Rankine) part of the Green function.

Because this Green function satisfies the linearized free-surface boundary conditions, the bottom boundary condition, and the far-field conditions, only integrals over the matching surface remain in the integral equation relating $\varphi$ and $\varphi_{r}$, 


$$
\begin{gathered}
-2 \pi \varphi(P, t)+\int_{S_{S}} \varphi(Q, t) G_{r}(P, Q, 0) \mathrm{d} S_{Q}-\int_{0}^{t} \mathrm{~d} \tau \int_{S_{S}} \varphi(Q, \tau) H_{r \tau}(P, Q, t-\tau) \mathrm{d} S_{Q} \\
=\int_{S_{S}} \varphi_{r}(Q, t) G(P, Q, 0) \mathrm{d} S_{Q}-\int_{0}^{t} \mathrm{~d} \tau \int_{S_{S}} \varphi_{r}(Q, \tau) H_{\tau}(P, Q, t-\tau) \mathrm{d} S_{Q}
\end{gathered}
$$

Here $P$ is assumed to lie on $S_{S}$.

The geometry of $S_{S}$ is chosen to be a vertical cylinder of radius $a$ and depth $h$ (as shown in Fig. 1) such that the variable of surface integration $Q$ may be expressed in cylindrical coordinates $Q=Q(a, \theta, z)$. For this geometry of $S_{S}$, the integral relation becomes:

$$
\begin{aligned}
& -2 \pi \varphi(P, t)+a \int_{0}^{2 \pi} \int_{-h}^{0} \varphi(a, \theta, z, t) G_{r}(P, a, \theta, z, 0) \mathrm{d} z \mathrm{~d} \theta-\int_{0}^{t}\left[a \int_{0}^{2 \pi} \int_{-h}^{0} \varphi(a, \theta, z, \tau) H_{r \tau}(P, a, \theta, z, t-\tau) \mathrm{d} z \mathrm{~d} \theta\right] \mathrm{d} \tau \\
& =a \int_{0}^{2 \pi} \int_{-h}^{0} \varphi_{r}(a, \theta, z, t) G(P, a, \theta, z, 0) \mathrm{d} z \mathrm{~d} \theta-\int_{0}^{t}\left[a \int_{0}^{2 \pi} \int_{-h}^{0} \varphi_{r}(a, \theta, z, \tau) H_{\tau}(P, a, \theta, z, t-\tau) \mathrm{d} z \mathrm{~d} \theta\right] \mathrm{d} \tau .
\end{aligned}
$$

\subsubsection{Time integrations}

The integrations with respect to time in Eq. 8 represent a convolution of the solution and the free-surface Green function. By assuming a form for the time variation of the potential (i.e., constant between time-steps), the velocity potential can be removed from the time integrals and integrals of the Green function become perfect integrals of a time derivative. The equation for the velocity potential at each new time-step becomes an integral equation in space only and depends on the solution of the velocity potential at all previous time-steps as follows:

$$
\begin{aligned}
& -2 \pi \varphi\left(P, t_{K}\right)+a \int_{0}^{2 \pi} \int_{-h}^{0} \varphi\left(\theta, z, t_{K}\right)\left[G_{r}(P, \theta, z, 0)+\gamma^{0}(P, \theta, z)\right] \mathrm{d} z \mathrm{~d} \theta \\
& -a \int_{0}^{2 \pi} \int_{-h}^{0} \varphi_{r}\left(\theta, z, t_{K}\right)\left[G(P, \theta, z, 0)+\lambda^{0}(P, \theta, z)\right] \mathrm{d} z \mathrm{~d} \theta \\
& =\sum_{k=1}^{K-1} a \int_{0}^{2 \pi} \int_{-h}^{0} \varphi\left(\theta, z, t_{k}\right) \gamma^{K-k}(P, \theta, z) \mathrm{d} z \mathrm{~d} \theta-\sum_{k=1}^{K-1} a \int_{0}^{2 \pi} \int_{-h}^{0} \varphi_{r}\left(\theta, z, t_{k}\right) \lambda^{K-k}(P, \theta, z) \mathrm{d} z \mathrm{~d} \theta,
\end{aligned}
$$

where

$$
\bar{\gamma}^{K, k}(P, \theta, z)=\frac{1}{\Delta t} \int_{t_{k-1}}^{t_{k}} H_{r}\left(P, \theta, z, t_{K}-\tau\right) \mathrm{d} \tau
$$

and

$$
\gamma^{K-k}(P, \theta, z)= \begin{cases}\bar{\gamma}^{K, k+1}(P, \theta, z)-\bar{\gamma}^{K, k}(P, \theta, z), & k<K \\ \bar{\gamma}^{K, K}(P, \theta, z), & k=K .\end{cases}
$$

The functions $\gamma^{K-k}(P, \theta, z)$ (and an analogously defined $\lambda^{K-k}(P, \theta, z)$ for $H\left(P, \theta, z, t_{K}-\tau\right)$ ) are hereafter called "shell-functions" because they are integrals in time of the Green function with the field point $P$ and source point $Q$ located on the "shell surface". 


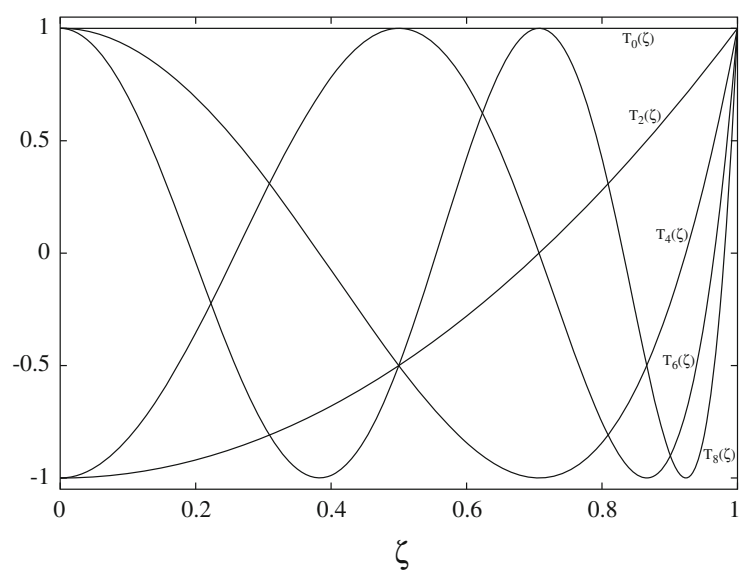

Fig. 2 Basis functions used to represent vertical variation of potential

\subsection{Spectral representation of the integral equation}

To construct a discrete approximation of the integral relation (9) numerically, it is necessary to represent the solution discretely in space and enforce the integral relation at a set of collocation points. The resulting linear system can be solved for the solution when a boundary condition is specified on $S_{S}$.

In this work, the geometry of the integration surface is exploited by representing the potential over the cylindrical shell in terms of orthogonal functions which are defined everywhere on the shell surface $S_{S}$. Chebyshev polynomials $\left[T_{j}(x)=\cos \left(j \cos ^{-1}(x)\right)\right]$ are used to represent variation in the vertical direction and Fourier components are used in the circumferential direction. This choice of global basis functions is a deviation from the usual piece-wise approximation of earlier works [9] and superior accuracy of the solution can be demonstrated. Additionally, because the surface $S_{s}$ is unchanging, the geometry and choice of basis function can be exploited in the numerical techniques used to evaluate the spatial integrals of the Green function as shown below.

\subsubsection{Discretization}

The representation of $\varphi$ on $S_{S}$ by the product of $N$ orthogonal polynomials in the circumferential direction and $J$ orthogonal polynomials in the vertical direction is summarized by the following equations.

$$
\begin{aligned}
& \varphi(\theta, z, t)=\sum_{j=0}^{J-1} \sum_{n=-N / 2}^{N / 2-1} \hat{\varphi}_{n j}(t) T_{2 j}\left(\frac{z}{h}+1\right) \mathrm{e}^{\mathrm{i} n \theta}, \\
& \hat{\varphi}_{n j}=\frac{2}{N J} \sum_{k=1}^{J} \sum_{m=0}^{N-1} \varphi\left(h\left(\zeta_{k}-1\right), \theta_{m}\right) T_{2 j}\left(\zeta_{k}\right) \mathrm{e}^{-\mathrm{i} n \theta_{m}},
\end{aligned}
$$

where $\theta_{m}=2 \pi m / N$ and $\zeta_{k}$ are the roots of $T_{2 J}\left(\zeta_{k}=\cos (\pi(2 k-1) / 4 J)\right)$. The prime on the first summation in (12) and (14) indicates that a factor of one half should be included when $j=0$.

In the vertical direction, only the even-ordered Chebyshev polynomials are used and a coordinate transformation $\left(\zeta=\frac{z}{h}+1\right)$ is used such that the argument of the Chebyshev polynomial always lies between zero and one. The use of these basis functions, which form an orthogonal set and are illustrated in Fig. 2, produces a solution that automatically satisfies the no-flux bottom boundary condition. Furthermore, the solution is expected to vary more rapidly near the free surface so it is appropriate to also concentrate the rapidly varying part of the basis function near the free surface.

A similar representation can be written for $\varphi_{r}$ : 
$\varphi_{r}(\theta, z, t)=\sum_{j=0}^{J-1} \sum_{n=-N / 2}^{N / 2-1} \hat{\varphi}_{r_{n j}}(t) T_{2 j}\left(\frac{z}{h}+1\right) \mathrm{e}^{\mathrm{i} n \theta}$,
$\hat{\varphi}_{r_{n j}}=\frac{2}{N J} \sum_{k=1}^{J} \sum_{m=0}^{N-1} \phi_{r}\left(h\left(\zeta_{k}-1\right), \theta_{m}\right) T_{2 j}\left(\zeta_{k}\right) \mathrm{e}^{-\mathrm{i} n \theta_{m}}$.

By denoting $\hat{\varphi}_{n j}^{K}=\hat{\varphi}_{n j}\left(t_{K}\right)$, the following relation between $\hat{\varphi}$ and $\hat{\varphi}_{r}$ at time $t_{K}$ is obtained:

$$
\begin{aligned}
& \sum_{j=0}^{J-1} \sum_{n=-N / 2}^{N / 2-1} \hat{\varphi}_{n j}^{K} T_{2 j}\left(\frac{z^{\prime}}{h}+1\right) \mathrm{e}^{\mathrm{i} n \theta^{\prime}}+a \sum_{j=0}^{J-1} \sum_{n=-N / 2}^{N / 2-1} \hat{\varphi}_{n j}^{K}\left[\hat{G}_{r_{n j}}(P)+\hat{\gamma}_{n j}^{0}(P)\right] \\
& \quad-a \sum_{j=0}^{J-1} \sum_{n=-N / 2}^{N / 2-1} \hat{\varphi}_{r_{n j}}^{K}\left[\hat{G}_{n j}(P)+\hat{\lambda}_{n j}^{0}(P)\right]=a \sum_{k=1}^{K-1} \sum_{j=0}^{J-1} \sum_{n=-N / 2}^{N / 2-1} \hat{\varphi}_{n j}^{k} \hat{\gamma}_{n j}^{K-k}(P)-a \sum_{k=1}^{K-1} \sum_{j=0}^{J-1} \sum_{n=-N / 2}^{N / 2-1} \hat{\varphi}_{r_{n j}}^{k} \hat{\lambda}_{n j}^{K-k}(P),
\end{aligned}
$$

where

$$
\begin{aligned}
& \hat{G}_{n j}(P)=\frac{1}{2 \pi} \int_{0-h}^{2 \pi} \int_{0}^{0} G(P, \theta, z, 0) T_{2 j}\left(\frac{z}{h}+1\right) \mathrm{e}^{\mathrm{i} n \theta} \mathrm{d} z \mathrm{~d} \theta, \\
& \hat{G}_{r_{n j}}(P)=\frac{1}{2 \pi} \int_{0}^{2 \pi} \int_{-h}^{0} G_{r}(P, \theta, z, 0) T_{2 j}\left(\frac{z}{h}+1\right) \mathrm{e}^{\mathrm{i} n \theta} \mathrm{d} z \mathrm{~d} \theta, \\
& \hat{\gamma}_{n j}^{K-k}(P)=\frac{1}{2 \pi} \int_{0-h}^{2 \pi} \int_{-h}^{0} \gamma^{K-k}(P, \theta, z) T_{2 j}\left(\frac{z}{h}+1\right) \mathrm{e}^{\mathrm{i} n \theta} \mathrm{d} z \mathrm{~d} \theta, \\
& \hat{\lambda}_{n j}^{K-k}(P)=\frac{1}{2 \pi} \int_{0}^{2 \pi} \int_{-h}^{0} \lambda^{K-k}(P, \theta, z) T_{2 j}\left(\frac{z}{h}+1\right) \mathrm{e}^{\mathrm{i} n \theta} \mathrm{d} z \mathrm{~d} \theta .
\end{aligned}
$$

Here $\hat{G}$ and $\hat{G}_{r}$ are coefficients of the impulsive (or Rankine) part of the Green function (i.e., the Green function evaluated at time equal zero) and $\hat{\gamma}$ and $\hat{\lambda}$ are coefficients related to the time-dependent part of the Green function as defined by (10) and (11). $\hat{\gamma}$ and $\hat{\lambda}$ are labeled "shell coefficients" because they are Fourier-Chebyshev coefficients of the shell-functions.

\subsubsection{Collocation}

In order to create a linear relationship between $\hat{\varphi}^{K}$ and $\hat{\varphi}_{r}^{K}$, the integral relation is asserted at a collection of points on the cylindrical surface $S_{s}$. Collocation points $P_{n^{\prime} j^{\prime}}$ are chosen as $N$ evenly spaced points in the circumferential direction and the first $J$ roots of $T_{2 J}\left(\frac{z}{h}+1\right)$ in the vertical direction. With these choices, a linear system results that can be written symbolically as follows:

$$
[\mathbf{S}+\mathbf{A}+\mathbf{C}] \hat{\varphi}^{K}-[\mathbf{B}+\mathbf{D}] \hat{\varphi}_{r}^{K}=\mathbf{E}^{K},
$$

where the matrices are composed of the following elements:

$S_{p q}=T_{2 j}\left(\zeta_{j^{\prime}}\right) \mathrm{e}^{\mathrm{i} n \theta_{n^{\prime}}}$

$A_{p q}=\hat{G}_{n j}\left(P_{n^{\prime} j^{\prime}}\right), \quad B_{p q}=\hat{G}_{r_{n j}}\left(P_{n^{\prime} j^{\prime}}\right), \quad C_{p q}=\hat{\gamma}_{n j}^{0}\left(P_{n^{\prime} j^{\prime}}\right), \quad D_{p q}=\hat{\lambda}_{n j}^{0}\left(P_{n^{\prime} j^{\prime}}\right)$,

and 


$$
E_{p}^{K}=a \sum_{k=1}^{K-1} \sum_{j=0}^{J-1} \sum_{n=-N / 2}^{N / 2-1} \hat{\varphi}_{n j}^{k} \hat{\gamma}_{n j}^{K-k}\left(P_{n^{\prime} j^{\prime}}\right)-a \sum_{k=1}^{K-1} \sum_{j=0}^{J-1} \sum_{n=-N / 2}^{N / 2-1} \hat{\Psi}_{n j}^{k} \hat{\lambda}_{n j}^{K-k}\left(P_{n^{\prime} j^{\prime}}\right) .
$$

The collocation-point mapping between point element $p$ and $n j$, and $q$ and $n^{\prime} j^{\prime}$ depends on the ordering of the rows and columns of collocation points in the matrices. Because the matrices are non-sparse, there is not an advantage to any particular ordering.

\subsection{Uniqueness and convolution of the Green function coefficients}

The total computational effort required to set up the linear system can be split into two contributions. The evaluation of the integrals in the Green-function coefficients (17-20) (which appear in the matrices (23-26)), and the convolution of the past solution with the Green-function coefficients (summations in $E_{p}$, Eq. 27). Because the $E_{p}^{K}$ vector must be computed anew at each time step, it is important to perform these summations as efficiently as possible. Both of these computational requirements can be reduced by exploiting the axi-symmetric geometry of the shell matching surface.

\subsubsection{Unique coefficients}

In traditional boundary-integral-equation formulations, the evaluation of the Green functions dominates the computational requirements of the formulation. Because the formulation presented here is intended to be a general solution of the outer flow that can be applied to a variety of internal problems, it is important to determine the most efficient way to compute and store the various coefficients for re-use.

Regardless of the Green function in question, when $P$ and $Q$ are expressed in cylindrical coordinates (see Fig. 3), the coefficients (17-20) are defined by integrals of the form:

$$
\hat{G}_{n j}\left(z^{\prime}, r^{\prime}, \theta^{\prime}\right)=\frac{1}{2 \pi} \int_{0}^{2 \pi}\left\{\int_{-h}^{0} G\left(r^{\prime}, z^{\prime}, a, \theta-\theta^{\prime}, z\right) T_{2 j}\left(\frac{z}{h}+1\right) \mathrm{d} z\right\} \mathrm{e}^{\mathrm{in} \theta} \mathrm{d} \theta .
$$

The shift property of the Fourier transform eliminates $\theta^{\prime}$ from the integrand

$$
\hat{G}_{n j}\left(z^{\prime}, r^{\prime}, \theta^{\prime}\right)=\mathrm{e}^{-\mathrm{i} n \theta^{\prime}} \frac{1}{2 \pi} \int_{0}^{2 \pi}\left\{\int_{-h}^{0} G\left(r^{\prime}, z^{\prime}, a, \theta, z\right) T_{2 j}\left(\frac{z}{h}+1\right) \mathrm{d} z\right\} \mathrm{e}^{\mathrm{i} n \theta} \mathrm{d} \theta=\mathrm{e}^{-\mathrm{i} n \theta^{\prime}} \hat{G}_{n j}\left(z^{\prime}, r^{\prime}, 0\right) .
$$

Thus, all required coefficients can be found easily from evaluations of $\left.\hat{G}_{(} z^{\prime}, r^{\prime}, 0\right)$, which is real because $P$ lies on the $x$-axis and the Green function will always be an even function with respect to $\theta$. This reduces the number of coefficients that need to be computed and stored by a factor of $2 N$. Because the plan is to pre-compute all of the shell-function coefficients, and store for future use and re-use, it is important to ensure that there are few enough unique coefficients that this is practical.

\subsubsection{Convolution}

Even with the coefficients pre-computed and stored, the computation required to perform the triple summations in (27) to compute $E_{p}^{K}$ can still be significant. Again, the axisymmetry of the geometry can be exploited to reduce the computational requirements. The convolution of Green-function integrals and the velocity-potential history appears in the vector $\mathbf{E}$ in the linear system (21).

$$
E_{p}^{K}=E_{n^{\prime} j^{\prime}}^{K}=a \sum_{k=1}^{k<K} \sum_{j=0}^{J-1} \sum_{n=-N / 2}^{N / 2-1} \hat{\varphi}_{n j}^{k} \hat{\gamma}_{n j}^{K-k}\left(P_{n^{\prime} j^{\prime}}\right)-a \sum_{k=1}^{K-1} \sum_{j=0}^{J-1} \sum_{n=-N / 2}^{N / 2-1} \hat{\Psi}_{n j}^{k} \hat{\lambda}_{n j}^{K-k}\left(P_{n^{\prime} j^{\prime}}\right) .
$$



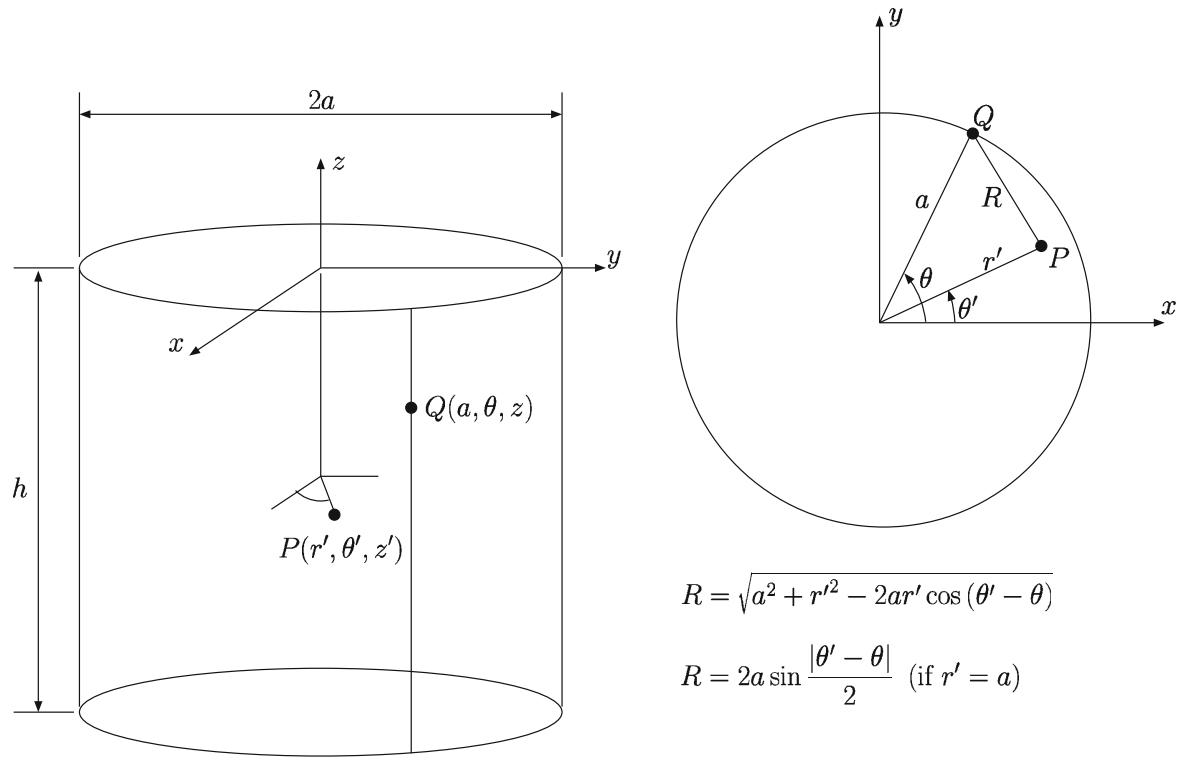

Fig. 3 Polar coordinate system for evaluation of the Green-function coefficients

Each term in this equation has the same character, so the first term involving summations of $\hat{\varphi}_{n j}^{k} \hat{\gamma}_{n j}^{K-k}\left(P_{n^{\prime} j^{\prime}}\right)$ will be denoted by $S_{1}$; the summations for the second term will follow in a similar manner. If we exploit the Hermitian symmetries in $n$ of the Green-function coefficients $\hat{\gamma}_{n j}^{K-k}$ and the solution $\hat{\varphi}_{n j}^{k^{r}}$, then

$$
S_{1}=\sum_{k=1}^{k<K} \sum_{j=0}^{J-1} \sum_{n=-N / 2}^{N / 2-1} \hat{\varphi}_{n j}^{k} \hat{\gamma}_{n j}^{K-k}\left(P_{n^{\prime} j^{\prime}}\right)=2 \sum_{k=1}^{k<K} \sum_{j=0}^{J-1} \sum_{n=0}^{N / 2-1}\left(\hat{\varphi}_{n j}^{k^{r}} \hat{\gamma}_{n j}^{K-k^{r}}+\hat{\varphi}_{n j}^{k^{i}} \hat{\gamma}_{n j}^{K-k^{i}}\right) \text {. }
$$

The superscripts ${ }^{r}$ and ${ }^{i}$ denote the real and imaginary parts of the complex values, respectively. With an eye toward pre-computing the shell coefficients $\hat{\gamma}$ and $\hat{\lambda}$, we note that, without taking advantage of any symmetries, the above summation must be performed at $N J$ collocation points $\left(P_{n^{\prime} j^{\prime}}\right)$, requiring $2 N^{2} J^{2} K$ floating-point multiplications to compute the entire vector $\mathbf{E}$ at the $K$ th time-step. These calculations involve $2 N^{2} J^{2} K$ unique coefficients that need to be evaluated. However, as illustrated by (29), the shift property of the Fourier transform relates the coefficients for field points $P_{n^{\prime} j^{\prime}}$ at a constant depth. All of the remaining unique coefficients are real, reducing the number of unique or distinctive coefficients by a factor of $2 N$ to $N J^{2} K$ :

$$
\hat{\gamma}_{n j}\left(P_{n^{\prime} j^{\prime}}\right)=\mathrm{e}^{-\mathrm{i} n^{\prime} \Delta \theta^{\prime}} \hat{\gamma}_{n j}\left(P_{0 j^{\prime}}\right)=\hat{\gamma}_{n j}\left(P_{0 j^{\prime}}\right) \cos \left(n^{\prime} \Delta \theta^{\prime}\right)-\mathrm{i} \hat{\gamma}_{n j}\left(P_{0 j^{\prime}}\right) \sin \left(n^{\prime} \Delta \theta^{\prime}\right) \text {. }
$$

Thus, the convolution summation becomes:

$$
\begin{aligned}
S_{1} & =\bar{E}_{n^{\prime} j^{\prime}}^{K}=2 \sum_{k=1}^{k<K} \sum_{j=0}^{J-1} \sum_{n=0}^{J / 2-1} \hat{\varphi}_{n j}^{k^{r}} \hat{\gamma}_{n j}^{K-k}\left(P_{0 j^{\prime}}\right) \cos \left(n^{\prime} \Delta \theta^{\prime}\right)-\hat{\varphi}_{n j}^{k^{i}} \hat{\gamma}_{n j}^{K-k}\left(P_{0 j^{\prime}}\right) \sin \left(n^{\prime} \Delta \theta^{\prime}\right) \\
& =2 \cos \left(n^{\prime} \Delta \theta^{\prime}\right) \sum_{k=1}^{k<K} \sum_{j=0}^{J-1} \sum_{n=0}^{N / 2-1} \hat{\varphi}_{n j}^{k^{r}} \hat{\gamma}_{n j}^{K-k}\left(P_{0 j^{\prime}}\right)-2 \sin \left(n^{\prime} \Delta \theta^{\prime}\right) \sum_{k=1}^{k<K} \sum_{j=0}^{J-1} \sum_{n=0}^{N / 2-1} \hat{\varphi}_{n j}^{k^{i}} \hat{\gamma}_{n j}^{K-k}\left(P_{0 j^{\prime}}\right) .
\end{aligned}
$$

Although $n^{\prime}$ does not appear inside the triple summation, $N J K$ multiplications are still needed to compute $\bar{E}_{n^{\prime} j^{\prime}}$. However, the bulk of this computation (the triple summations in (33)) can be re-used for all $N$ values of $n^{\prime}$ for each value of $j^{\prime}$. Therefore, the total number of floating-point multiplications required to evaluate the entire vector $\mathbf{E}$ is reduced to $2 N J^{2} K$. 


\section{Computation of coefficients of Green functions}

The time-dependent free-surface Green function represents the potential at time $t$ due to a source that comes into existence at time $\tau$. This section presents the details of the evaluation of the various free-surface Green functions used in the formulation above, simple-source, dipole and the unsteady free-surface Green functions in infinite and finite depth fluid.

The simple-source Green function and the dipole Green function are singular but the singularity is integrable. Newman [10] presents methods to evaluate integrals of the simple source and dipole over flat panels with linear variation in source strength or dipole moment. For higher-order panel methods, coordinate-transform methods are usually used to cancel the singularity and allow Gaussian quadrature to be efficient [11]. The spectral shell method presents a special case in which integration of a source distribution weighted by Fourier-Chebyshev basis functions needs to be carried over a cylinder. Direct numerical integration of the source or dipole is inefficient and has difficulties with the singular nature of the source and dipole. Section 3.1 below presents a semi-analytic procedure for evaluating these singular integrals.

The unsteady free-surface Green functions are defined by integration of an oscillatory integrand. Numerically evaluation of this integral can be tricky; Beck [12] presents detailed methods relying on asymptotic and series expansions of the integrand for evaluating the infinite-depth free-surface Green function and Newman [13] tackles the finite-depth case. In these studies, the emphasis is on evaluating the Green function for a specific source and field point and relying on numerical integration to perform the needed integrals over each boundary element. Our specific choice of shell geometry and basis function allow analytical integrations of the Green functions in space and time to be performed; doing this changes the Green functions to something different from the forms previously studied in the literature. Fortunately, the analytical integration step truncates the oscillatory tail of the integrands sufficiently to allow the use of more direct numerical integrations over wave-number. Sections 3.2 and 3.5 below present these techniques for the infinite-depth case and finite-depth case, respectively.

\subsection{Simple-source Green function}

The boundary-integral-equation method for the interior problem uses a simple source defined as the reciprocal of the distance between the field and source points;

$$
\begin{aligned}
& G^{s}(P, Q)=\frac{1}{|P Q|}, \\
& G^{s}\left(r^{\prime}, z^{\prime}, a, z, \theta\right)=\frac{1}{\sqrt{R^{2}+\left(z-z^{\prime}\right)^{2}}},
\end{aligned}
$$

where $R$ is the radial direction between $P$ and $Q$ (see Fig. 3). Fourier-Chebyshev coefficients of the derivative of $G^{s}$ with respect to $Q$ moving in the direction normal to $S_{S}$, (i.e., with respect to $a$ ) are also required.

$$
\begin{aligned}
G_{v}^{s} & =\frac{\partial}{\partial n_{Q}}\left[G^{s}\right]=\frac{\partial}{\partial a}\left[G^{s}\right]=\frac{\partial}{\partial R}\left[G^{s}\right] \frac{\partial R}{\partial a} \\
& =-\frac{\partial R}{\partial a} \frac{R}{\left[R^{2}+\left(z-z^{\prime}\right)^{2}\right]^{3 / 2}} .
\end{aligned}
$$

The required integrations of this source Green function may be evaluated efficiently and accurately by first performing the integration in the vertical direction, followed by integration in the circumferential direction. The result of the vertical integration is a function that is only logarithmically singular at $\theta=0$. The strength of this singularity is found analytically and subtracted so that the regular remainder can be integrated numerically. The Fourier series for the singular part is found analytically to complete the evaluation. 


\subsubsection{Integration of $G^{s}$ and $G_{v}^{s}$ in the vertical direction}

The integrals needed are:

$$
\begin{gathered}
\bar{G}_{j}^{s}=\int_{-h}^{0} G^{s} T_{2 j}\left(\frac{z}{h}+1\right) \mathrm{d} z=\int_{-h}^{0} \frac{T_{2 j}\left(\frac{z}{h}+1\right)}{\sqrt{R^{2}+\left(z-z^{\prime}\right)^{2}}} \mathrm{~d} z=\int_{0}^{1} \frac{T_{2 j}(\zeta)}{\sqrt{\left(\frac{R}{h}\right)^{2}+\left(\zeta^{\prime}-\zeta\right)^{2}}} \mathrm{~d} \zeta, \\
\bar{G}_{v_{j}}^{s}=\int_{-h}^{0} G_{v}^{s} T_{2 j}\left(\frac{z}{h}+1\right) \mathrm{d} z=\int_{-h}^{0} \frac{-R R_{a} T_{2 j}\left(\frac{z}{h}+1\right)}{\left[R^{2}+\left(z-z^{\prime}\right)^{2}\right]^{3 / 2}} \mathrm{~d} z=\int_{0}^{1} \frac{-R R_{a} T_{2 j}(\zeta)}{\left[\left(\frac{R}{h}\right)^{2}+\left(\zeta^{\prime}-\zeta\right)^{2}\right]^{3 / 2}} \mathrm{~d} \zeta,
\end{gathered}
$$

where a change of variable $\zeta=\frac{z}{h}+1$ (and $\zeta^{\prime}=\frac{z^{\prime}}{h}+1$ ) normalizes the range of integration. Examination of these integrals indicates that the integrands are singular at $z^{\prime}=z$ when $R=0$. These singularities are non-integrable. In (39), inclusion of the $R R_{a}$ factor in the integrand cancels the singularity sufficiently, rendering $\bar{G}_{v_{j}}^{s}$ finite for all values of $R$. For $\bar{G}_{j}^{s}$, Eq. 38 is infinite for $R=0$ but it will be shown that the behavior of $\bar{G}_{j}^{s}$ for small $R$ is that of a logarithmic singularity $-2 T_{2 j}\left(\frac{z^{\prime}}{h}+1\right) \log (R)$. Thus $\bar{G}_{j}^{s}$ is integrable in the evaluation of the Fourier-Chebyshev coefficient $\hat{G}_{v}^{s}$. Additionally, knowledge of this singular behavior provides a convenient way of smoothing $\bar{G}_{j}^{s}$ by subtracting the singular part. The behavior of $\bar{G}_{j}^{s}$ at $R=0$ can be most easily determined by writing $T_{j}(x)$ as a polynomial,

$$
T_{j}(x)=\sum_{k=0}^{j} a_{k} x^{k}
$$

and integrating analytically term by term:

$$
\bar{G}_{j}^{s}=\sum_{k=0}^{j} a_{k} \int_{0}^{1} \frac{\zeta^{k}}{\sqrt{\left(\frac{R}{h}\right)^{2}+\left(\zeta^{\prime}-\zeta\right)^{2}}} \mathrm{~d} \zeta .
$$

For $k=0$,

$$
I_{0}=\int_{0}^{1} \frac{1}{\sqrt{\left(\frac{R}{h}\right)^{2}+\left(\zeta^{\prime}-\zeta\right)^{2}}} \mathrm{~d} \zeta=\log \left[\frac{h^{2}\left(\sqrt{\left(\frac{R}{h}\right)^{2}+\left(\zeta^{\prime}-1\right)^{2}}-\left(\zeta^{\prime}-1\right)\right)\left(\sqrt{\left(\frac{R}{h}\right)^{2}+\zeta^{\prime 2}}+\zeta^{\prime}\right.}{R^{2}}\right]
$$

For $K>0$,

$$
I_{k}=\int_{0}^{1} \frac{\zeta^{k}}{\sqrt{\left(\frac{R}{h}\right)^{2}+\left(\zeta^{\prime}-\zeta\right)^{2}}} \mathrm{~d} \zeta=\frac{1}{k} \sqrt{\left(\frac{R}{h}\right)^{2}+\left(\zeta^{\prime}-1\right)^{2}}+\frac{(2 k-1) \zeta^{\prime}}{k} I_{k-1}-\frac{k-1}{k}\left(\left(\frac{R}{h}\right)^{2}+\zeta^{\prime 2}\right) I_{k-2}
$$

These equations provide a recursive relation for the magnitude of the logarithmic singularity for each power of $k$.

$$
C_{k}=\frac{(2 k-1) \zeta^{\prime}}{k} C_{k-1}-\frac{k-1}{k}\left(\left(\frac{R}{h}\right)^{2}+\zeta^{\prime 2}\right) C_{k-2}, \quad C_{0}=1 .
$$

In the limit $R \rightarrow 0$, this recursive relation may be easily solved by inspection to give $C_{k}=\left(\zeta^{\prime}\right)^{k}$. Thus, the magnitude of the logarithmic singularity is

$$
\sum_{k=0}^{j} a_{k}\left(\zeta^{\prime}\right)^{k}=T_{2} j\left(\zeta^{\prime}\right)=T_{2} j\left(\frac{z^{\prime}}{h}+1\right)
$$


As expected, the strength of the singularity depends on the $z$-coordinate of the field point, being half as strong if $z^{\prime}$ is equal to 0 or $-h$. In summary, the singular behavior of $\bar{G}_{j}^{s}$ is,

$$
\left(\bar{G}_{j}^{s}\right)_{\text {sing }}= \begin{cases}0 & z^{\prime}>0 \text { or } z^{\prime}<-h, \\ -T_{2 j}\left(\frac{z^{\prime}}{h}+1\right) \log [R] & z^{\prime}=0 \text { or } z^{\prime}=h, \\ -2 T_{2 j}\left(\frac{z^{\prime}}{h}+1\right) \log [R] & -h<z^{\prime}<0 .\end{cases}
$$

The above analytical integrations suggest a term-by-term method of computing the required integrals; however, the series is very poorly conditioned so numerical integration is a better choice. A change of variable $\zeta=\cos (y)$ casts the integrals into a form well handled by Filon integration:

$$
\begin{aligned}
\bar{G}_{j}^{s} & =\int_{0}^{\pi / 2} \frac{\sin y}{\sqrt{\left(\frac{R}{h}\right)^{2}+\left(\zeta^{\prime}-\cos y\right)^{2}}} \cos (2 j y) \mathrm{d} y, \\
\bar{G}_{v_{j}}^{s} & =\int_{0}^{\pi / 2} \frac{\sin y}{\left[\left(\frac{R}{h}\right)^{2}+\left(\zeta^{\prime}-\cos y\right)^{2}\right]^{3 / 2}} \cos (2 j y) \mathrm{d} y .
\end{aligned}
$$

A FFT-based Filon integration procedure is used to efficiently compute the integrals for all $J$ values of $j$ with one FFT operation [14, Sect. 13.9].

\subsubsection{Fourier coefficients of $\bar{G}_{j}^{s}$ and $\bar{G}_{v_{j}}^{s}$}

The integration over $\theta$ is equivalent to finding the Fourier coefficients of $\bar{G}_{j}^{s}$ and $\bar{G}_{v_{j}}^{s}$. Defining

$$
\left(\bar{G}_{j}^{s}\right)_{\text {reg }}=\bar{G}_{j}^{s}-\left(\bar{G}_{j}^{s}\right)_{\text {sing }}
$$

smooths the singular behavior. Additionally, it is clear that $\left(\bar{G}_{j}^{s}\right)_{\text {reg }}$ and $\bar{G}_{v_{j}}^{s}$ are even functions of $\theta$, hence the resulting Fourier coefficients will be real. The smoothness of these functions allows efficient computation of $\left(\hat{G}_{j}^{s}\right)_{\text {reg }}$ and $\hat{G}_{v_{j}}^{s}$ by FFT. To complete the evaluation of $\hat{G}_{j}^{s}$, the Fourier series representing the singular part is needed. This series is found analytically to be

$$
\log [R]=\log \left[2 a \sin \frac{|\theta|}{2}\right]=\log [a]+\sum_{k=1}^{\infty} \frac{-1}{k} \cos k \theta .
$$

\subsection{Unsteady free-surface Green function-infinite depth}

The unsteady free-surface Green function used in the time-dependent formulation represents the velocity potential at $P$ in a fluid of infinite depth with a free surface due to a source disturbance at $Q$. The Green function represents the potential at time $t$ due to this disturbance introduced at time $\tau$ that satisfies the Laplace equation and the boundary conditions:

$$
\nabla^{2} G(P, Q, t-\tau)=\delta(P-Q), \quad G_{\tau \tau}+\left.G_{z}\right|_{z=0}=0 .
$$

Such a function is given by Finkelstein [15] as:

$$
G(P, Q, t-\tau)=\frac{1}{r}-\frac{1}{r^{\prime}}+2 \int_{0}^{\infty} \mathrm{d} k \mathrm{e}^{(z+\zeta) k}\{1-\cos \sqrt{k}(t-\tau)\} J_{0}(k R),
$$


where

$$
R=\sqrt{(x-\xi)^{2}+(y-\eta)^{2}}, \quad r=\sqrt{R^{2}+(z-\zeta)^{2}}, \quad r^{\prime}=\sqrt{R^{2}+(z+\zeta)^{2}}
$$

and $J_{0}$ is the Bessel function of the first kind and of order zero, and $R$ is the horizontal distance between $P$ and $Q$. This Green function can be separated into an impulsive, or Rankine, part and a time-dependent part:

$$
G(P, Q, t-\tau)=\left(\frac{1}{r}-\frac{1}{r^{\prime}}\right)+H(P, Q, t-\tau)
$$

The coefficients of the impulsive part are evaluated using the methods mentioned in the previous section for the simple-source Green function. Integrals with respect to $\tau$ of the time-dependent part are needed in the integral-equation formulation. Performing this integration analytically:

$$
\begin{aligned}
\bar{H}^{K-k}(P, Q)= & \int_{t_{k-1}}^{t_{k}} H\left(P, Q, t_{K}-\tau\right) \mathrm{d} \tau=2\left(t_{k}-t_{k-1}\right) \int_{0}^{\infty} \mathrm{e}^{(z+\zeta) k} J_{0}(k R) \mathrm{d} k \\
& +\left.2 \int_{0}^{\infty} \mathrm{e}^{(z+\zeta) k} \frac{1}{\sqrt{k}} \sin \sqrt{k}(t-\tau) J_{0}(k R) \mathrm{d} k\right|_{t_{k-1}} ^{t_{k}} .
\end{aligned}
$$

The first integral can be identified as $\frac{1}{r^{\prime}}$ by replacing the Bessel function by its integral representation and reversing the orders of integration. Thus the methods of the last section apply to the first integral. The second integral needs to be evaluated numerically. Fortunately, the time integration has increased the rate of decay of the integrand.

When the source point is at $r=a$ and $\theta^{\prime}=0$, the coefficients of the time-dependent part are:

$$
\hat{H}_{n j}\left(z^{\prime}, a, t-\tau\right)=\frac{1}{2 \pi} \int_{0}^{2 \pi} \int_{-h}^{0} \bar{H}(P, Q, t-\tau) T_{2 j}\left(\frac{z}{h}+1\right) \mathrm{e}^{-\mathrm{i} n \theta} \mathrm{d} z \mathrm{~d} \theta .
$$

Evaluation of the coefficients for the time-dependent part proceeds in a different manner than the simple source and dipole. Because of the highly oscillatory nature of $H$ with respect to $R$, discrete Fourier transforms are not feasible. Instead, the order of integrations in equation (55) is reversed; the integration with respect to $\theta$ is performed first by taking advantage of basic Bessel function identities. These Fourier coefficients are then integrated numerically in the vertical direction.

\subsection{Fourier coefficients of $\bar{H}$ and $\bar{H}_{v}$}

As noted, the $1 / r^{\prime}$ part of (55) is treated using the methods of the previous section, this section treats the circumferential integration of the second integral, denoted here $\bar{I}$. By referring to Fig. 3 , the 'addition formula' for $J_{0}$ is seen to be:

$$
J_{0}(k R)=J_{0}\left(k r_{1}\right) J_{0}(k a)+2 \sum_{m=1}^{\infty} J_{m}\left(k r_{1}\right) J_{m}(k a) \cos (m \theta) .
$$

Multiplication of both sides by $\cos n \theta$ and integrating from 0 to $2 \pi$ provides the needed integrals:

$$
\begin{aligned}
& \frac{1}{2 \pi} \int_{0}^{2 \pi} J_{0}(k R) \cos n \theta \mathrm{d} \theta=J_{n}\left(k r_{1}\right) J_{n}(k a), \\
& \frac{1}{2 \pi} \int_{0}^{2 \pi} \frac{\partial}{\partial a} J_{0}(k R) \cos n \theta \mathrm{d} \theta=k J_{n}\left(k r_{1}\right) J_{n}^{\prime}(k a) .
\end{aligned}
$$


Multiplication of (56) by $\sin n \theta$ and integrating confirms that the Fourier coefficients are purely real. When $r_{1}=a$, which is the primary interest, the Fourier coefficients of $\bar{I}, \bar{I}_{v}$ are:

$$
\begin{aligned}
& \tilde{I}_{n}(a, z, \zeta, t-\tau)=\frac{1}{2 \pi} \int_{0}^{2 \pi} \bar{I} \cos (n \theta) \mathrm{d} \theta=2 \int_{0}^{\infty} \mathrm{e}^{\left(z^{\prime}+z\right) k} \frac{1}{\sqrt{k}} \sin \sqrt{k}(t-\tau) J_{n}^{2}(k) \mathrm{d} k, \\
& \tilde{I}_{v_{n}}(a, z, \zeta, t-\tau)=\frac{1}{2 \pi} \int_{0}^{2 \pi} \bar{I}_{v} \cos (n \theta) \mathrm{d} \theta=2 \int_{0}^{\infty} \mathrm{e}^{\left(z^{\prime}+z\right) k} \sqrt{k} \sin \sqrt{k}(t-\tau) J_{n}(k) J_{n}^{\prime}(k) \mathrm{d} k .
\end{aligned}
$$

The circumferential integrations introduce another Bessel function into the integrand which decays like $\frac{1}{\sqrt{k R}}$ for large argument, increasing the rate of decay. The differentiation with respect to the normal at the field point unfortunately decreases the rate of decay of that integrand, (Eq. 60). However, it does still decay and when the field point or source point is submerged, the exponential decay dominates in any case. For numerical evaluation, a change of variable is used to cast the problem into a form handled by standard Filon integration [14, Sect. 13.9].

\subsection{Integration of $\tilde{H}$ and $\tilde{H}_{v}$ in the vertical direction}

As $z$ varies, $\tilde{I}$ and $\tilde{I}_{v}$ are very well behaved and present no problems to numerical integration in the vertical direction. However, because the Chebyshev polynomials vary rapidly, a change of variable and Filon integration is used to evaluate the integrals efficiently [14, Sect. 13.9]:

$$
\begin{aligned}
& \hat{I}_{n j}(a, t-\tau)=\int_{0}^{\pi / 2} \tilde{I}_{n}\left(a, z^{\prime}, \cos y, t-\tau\right) \sin y \cos (2 j y) \mathrm{d} y, \\
& \hat{I}_{v_{n j}}(a, t-\tau)=\int_{0}^{\pi / 2} \tilde{I}_{v_{n}}\left(a, z^{\prime}, \cos y, t-\tau\right) \sin y \cos (2 j y) \mathrm{d} y .
\end{aligned}
$$

\subsection{Unsteady free-surface Green function-finite depth}

In finite-depth water, the unsteady free-surface Green function has a similar form to the infinite-depth case and satisfies a no leak condition on the bottom surface as well as the free-surface condition.

$$
\nabla^{2} G(P, Q, t-\tau)=\delta(P-Q), \quad G_{\tau \tau}+\left.G_{z}\right|_{z=0}=0,\left.\quad G_{z}\right|_{z=-h}=0 .
$$

Such a function is given by Finkelstein [7] as the sum of a Rankine part and a time-dependent part:

$$
G(P, Q, t-\tau)=G^{r}(P, Q)+H(P, Q, t-\tau),
$$

where $G^{r}$ is the Rankine part of the Green function,

$$
G^{r}(P, Q)=\frac{1}{r}+\frac{1}{r^{\prime \prime}}-2 \int_{0}^{\infty} \mathrm{e}^{-k h} \frac{\cosh k\left(z^{\prime}+h\right) \cosh k(z+h)}{\cosh k h} J_{0}(k R) \mathrm{d} k .
$$

Evaluation of this function is straightforward; the source and image parts are treated as in Sect. 3.1 and the integral part is easily summed using standard numerical integration. The integrand is regular and decays quickly enough, especially after the needed integration in the circumferential direction is performed analytically as in the infinitedepth case of the previous section. 
The time-dependent part $H$ is given by:

$$
H(P, Q, t-\tau)=2 \int_{0}^{\infty} \frac{\cosh k\left(z^{\prime}+h\right) \cosh k(z+h)}{\sinh k h \cosh k h}\{1-\cos \omega(k, h)(t-\tau)\} J_{0}(k R) \mathrm{d} k,
$$

where $\omega(k, h)=\sqrt{k \tanh k h}$. Integrals with respect to $\tau$ are needed in the formulation; performing this integration analytically results in:

$$
\begin{aligned}
\bar{H}^{K-k}= & \int_{t_{k-1}}^{t_{k}} H\left(P, Q, t_{K}-\tau\right) \mathrm{d} \tau=2\left(t_{k}-t_{k-1}\right) \int_{0}^{\infty} \frac{\cosh k\left(z^{\prime}+h\right) \cosh k(z+h)}{\sinh k h \cosh k h} J_{0}(k R) \mathrm{d} k \\
& +\left.2 \int_{0}^{\infty} \frac{\cosh k\left(z^{\prime}+h\right) \cosh k(z+h)}{\omega(k, h) \sinh k h \cosh k h} \sin \omega(k, h)\left(t_{K}-\tau\right) J_{0}(k R) \mathrm{d} k\right|_{t_{k-1}} ^{t_{k}} .
\end{aligned}
$$

The non-oscillatory part of each integrand in (67) is singular like $\frac{1}{k}$ near $k=0$. In each integral, this singularity is non-integrable, but $H$ remains finite because the singular parts cancel in the addition. Replacing $\sin [\omega(k, h)(t-\tau)]$ with a small-argument expansion demonstrates that the contribution to $\bar{H}$ of the neighborhood of $k \rightarrow 0$ is zero. However, for numerical evaluation, trigonometry relations are used to combine the two integrals into one with a finite integrand at $k=0$. This procedure is effective for all of the needed Green functions but is not necessary for derivatives of $\bar{H}$ with respect to $v$ and for nonzero Fourier modes because in these cases the integrands are always finite after integration in the circumferential direction. In any case, the techniques for evaluating the Fourier-Chebyshev coefficients of the finite depth Green function follow from (67) in the same manner as the infinite depth case presented in the previous section.

\section{Matching shell solution to viscous inner flows}

The generalized solution of the outer inviscid flow described above is applied as a boundary condition to an interior viscous-flow solution here. Application of the shell solution to inviscid problems in the interior region has been done previously and does not present any fundamental problems [9]. In contrast, the matching between an inviscid and a viscous solution represents a change of field equation across the boundary and careful attention must be paid to the meaning of the boundary condition that the exterior inviscid flow presents to the interior flow. In the examples here the interior viscous flow is solved via a pseudo-spectral solution technique of the Navier-Stokes equations [16].

\subsection{Interior-region viscous problem formulation}

Figure 4 illustrates the viscous-inviscid domain decomposition employed with the shell surface. The viscous region near the body is denoted $R_{1}$ and the inviscid region in the far field is region $R_{2}$.

For a fluid of uniform density $\rho$, conservation of mass can be written as

$$
\nabla \cdot \mathbf{u}(\mathbf{x}, t)=0,
$$

where the vector $\mathbf{u}$ represents the fluid velocity at the position $\mathbf{x}$ and time $t$. Conservation of momentum is similarly written as:

$$
\frac{\partial \mathbf{u}(\mathbf{x}, t)}{\partial t}+\mathbf{u} \cdot \nabla \mathbf{u}=\frac{1}{\rho}[-\nabla P(\mathbf{x}, t)+\nabla \cdot \underline{\underline{\tau}}]
$$




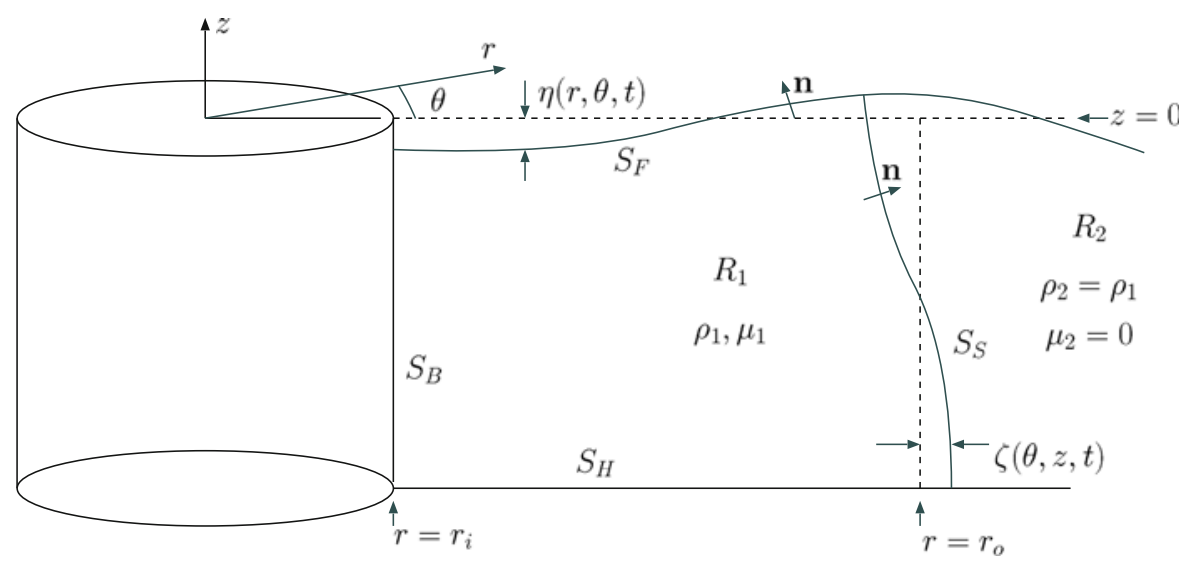

Fig. 4 Schematic of viscous and inviscid regions with free surface, with details shown in $\theta=0$ plane

where $P$ is the dynamic pressure in the fluid and $\underline{\underline{\tau}}$ is the viscous-stress tensor which represents traction forces on the boundary of an arbitrary fluid volume:

$P=p-\rho g z$.

The viscous region is bounded by four surfaces $S_{B}, S_{H}, S_{F}$, and $S_{S}$. No-slip boundary conditions are applied to the body and bottom surfaces while more complicated kinematic and dynamic boundary conditions are required for the fluid-atmosphere and shell-matching surface. For an arbitrary surface $S$ defined by the equation $F(r, \theta, z, t)=0$, the exact kinematic boundary condition that applies may be written in cylindrical coordinates as:

$u \frac{\partial F}{\partial r}+\frac{v}{r} \frac{\partial F}{\partial \theta}+w \frac{\partial F}{\partial z}=-\frac{\partial F}{\partial t}$.

Wehausen and Laitone [7] derive the appropriate conditions for the fluid-atmosphere interface $\left(S_{F}\right)$ by asserting that on the intersection between the fluid and atmosphere above, the normal component of stress must be continuous across the interface and any discontinuity in shear stress must be proportional to the surface curvature and surface tension. When shear stress and surface tension are neglected, the dynamic boundary conditions may be written in terms of the stress tensor $\underline{\underline{\mathbf{s}}}$ as follows:

$\underline{\underline{\mathbf{s}}} \mathbf{n}=\bar{p} \mathbf{n}=(\rho g z+\bar{P}) \mathbf{n}$.

Here, a prescribed pressure on the surface $S$ has been decomposed into a hydrostatic component $(\rho g z)$ and a dynamic component $(\bar{P})$.

\subsubsection{Boundary-condition linearization}

The equations for the kinematic (71) and dynamic (72) boundary conditions on an arbitrary, friction-less, surface will now be used to develop linearized boundary conditions on both the fluid-atmosphere interface $\left(S_{F}\right)$, and the viscous-inviscid interface $\left(S_{S}\right)$. The linearization procedure used is a perturbation technique in which a parameter $\epsilon$ is chosen that characterizes some feature of the motion that is considered "small". This allows the flow variables to be expanded in a power series in $\epsilon$ as follows:

$$
\begin{aligned}
& \mathbf{u}(r, \theta, z, t)=\epsilon \mathbf{u}^{(1)}+\epsilon^{2} \mathbf{u}^{(2)}+\cdots, \\
& P(r, \theta, z, t)=\epsilon P^{(1)}+\epsilon^{2} P^{(2)}+\cdots .
\end{aligned}
$$

Insertion of these expansions into the governing equations, and grouping like powers of $\epsilon$ results in a series of equations for each order of the solution. For $\epsilon^{1}$, the equation for conservation of momentum becomes,

$$
\frac{\partial \mathbf{u}^{(1)}}{\partial t}=-\frac{1}{\rho} \nabla P^{(1)}+\mu \nabla^{2} \mathbf{u}^{(1)} .
$$




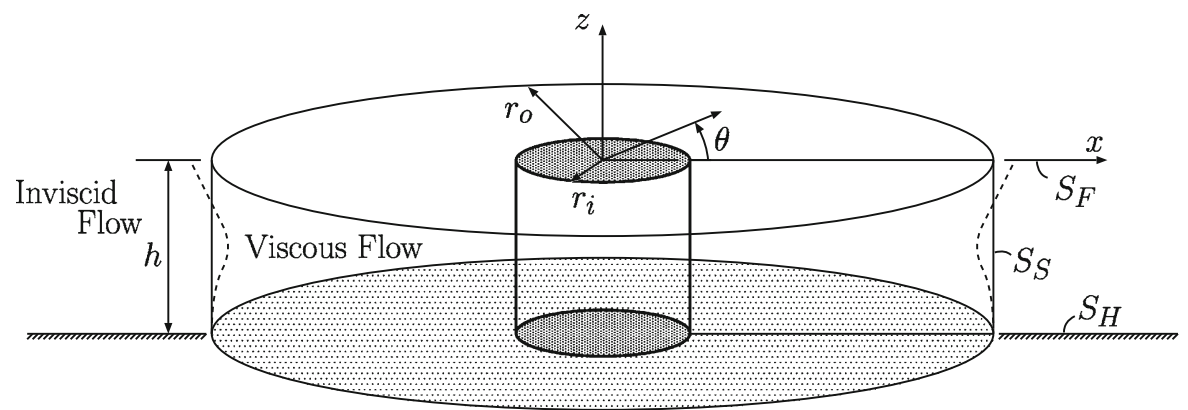

Fig. 5 Schematic of viscous interior-flow problem

To perform the linearization of the boundary conditions on the fluid-atmosphere interface, let the surface $S_{F}$ be described by the function $F_{f}(r, \theta, z, t)=z-\eta(r, \theta, t)=0$. With this choice, $\eta$ is expanded in powers of $\epsilon$.

$\eta(r, \theta, t)=\eta^{(0)}+\epsilon \eta^{(1)}+\epsilon^{2} \eta^{(2)}+\cdots$.

Insertion of these expansions into the kinematic boundary condition (71) and grouping of like powers of $\epsilon$ leads to a condition for $\mathbf{u}^{(1)}$ to be applied at the equilibrium position of the fluid-atmosphere interface (i.e., $\left.\eta^{(0)}=0\right)$ gives $w^{(1)}(r, \theta, 0, t)=\frac{\partial \eta^{(1)}}{\partial t}$.

The dynamic boundary condition on the free surface become:

$$
\left.\begin{array}{l}
\frac{\partial w^{(1)}}{\partial r}+\frac{\partial u^{(1)}}{\partial z}=0 \\
\frac{\partial v^{(1)}}{\partial z}+\frac{1}{r} \frac{\partial w^{(1)}}{\partial \theta}=0 \\
P^{(1)}-\rho g \eta^{(1)}-2 \mu \frac{\partial w^{(1)}}{\partial z}=\bar{P}^{(1)}
\end{array}\right\} \quad \text { at } z=0 .
$$

The term involving $g$ is a result of the use of dynamic, instead of the absolute pressure and results in a coupling between the kinematic and dynamic boundary conditions for the $\epsilon^{1}$ solution.

\subsubsection{Viscous-inviscid interface}

The linearization at the viscous-inviscid fluid interface $\left(S_{S}\right)$ is done in a similar manner. Let this surface be defined by the function $F_{S}(\theta, z, t)=r-\zeta(\theta, t)=0$. Expanding $\zeta$ in powers of $\epsilon$ gives:

$\zeta(\theta, z, t)=\zeta^{(0)}+\epsilon \zeta^{(1)}+\epsilon^{2} \eta^{(2)}+\cdots$

Choosing $\zeta^{(0)}=r_{o}$ specifies that the linearization is about $r_{o}$ and the kinematic boundary condition for the $\epsilon^{1}$ solution is:

$\frac{\partial \zeta^{(1)}}{\partial t}=u^{(1)}, \quad$ at $r=r_{o}$

and the dynamic boundary conditions become (Fig. 5):

$$
\left.\begin{array}{l}
P^{(1)}-2 \mu \frac{\partial u}{\partial r}=\bar{P}^{(1)} \\
r \frac{\partial}{\partial r}\left(\frac{v}{r}\right)+\frac{1}{r} \frac{\partial u}{\partial \theta}=0 \\
\frac{\partial w}{\partial r}+\frac{\partial u}{\partial z}=0
\end{array}\right\} \quad \text { at } r=r_{o} .
$$

Unlike the fluid-atmosphere interface, the kinematic condition and dynamic conditions for $\epsilon^{1}$ terms are uncoupled; $\zeta^{(1)}$ does not appear in the dynamic boundary conditions as $\eta^{(1)}$ did in (78). 


\subsection{Numerical solution of the Navier-Stokes equations}

In order to obtain an accurate numerical solution of the Navier-Stokes equations in the interior region, a pseudo-spectral, fractional-step method of solution is employed. A time-stepping method for the solution of the Navier-Stokes equations is used to solve for the velocity and pressure field in the viscous domain at a sequence of time-steps $t_{k}=k \Delta t,(k=1,2, \ldots)$. In this fractional-step method, an intermediate velocity field is found that satisfies the Navier-Stokes equation with the pressure terms removed. To complete each step, a pressure field is then found that corrects the intermediate velocity field to form the velocity and pressure field at the new time-step. This is a method that falls into the class of projection methods proposed by Chorin [17] and developed for use with free-surface flows by Yeung [18] in two dimensions and by Yu $[16,19]$ in three dimensions.

A time-difference scheme between the old $(K-1)$ and new $(K)$ time-steps provides,

$$
\begin{aligned}
& \frac{1}{\Delta t}\left(\mathbf{u}^{K}-\mathbf{u}^{K-1}\right)=\mathcal{Q}\left[-(\mathbf{u} \cdot \nabla) \mathbf{u}+\frac{1}{\operatorname{Re}} \tilde{\nabla}^{2} \mathbf{u}\right]-\nabla P^{K}, \\
& \nabla \cdot \mathbf{u}^{K}=0,
\end{aligned}
$$

where $\mathcal{Q}$ is a suitably defined difference operator. To solve these equations numerically, an intermediate velocity field ( $\hat{\mathbf{u}})$ is introduced which satisfies the momentum equation (82) without the pressure terms:

$$
\frac{1}{\Delta t}\left(\hat{\mathbf{u}}-\mathbf{u}^{K-1}\right)=\mathcal{Q}\left[-(\mathbf{u} \cdot \nabla) \mathbf{u}+\frac{1}{\operatorname{Re}} \tilde{\nabla}^{2} \mathbf{u}\right] .
$$

When this is subtracted from (82), an equation for the pressure field results:

$$
\frac{1}{\Delta t}\left(\mathbf{u}^{K}-\hat{\mathbf{u}}\right)=-\nabla P^{K} \text {. }
$$

The divergence of this equation, along with the continuity equation (83), provides a Poisson equation for the pressure field:

$$
\nabla^{2} P^{K}=\frac{1}{\Delta t} \nabla \cdot \hat{\mathbf{u}}
$$

Use of the continuity equation ensures mass conservation in the numerical scheme.

To summarize, an intermediate velocity field $\hat{\mathbf{u}}$ is solved for from a modified equation that neglects the pressure field (84). A Poisson equation for pressure field (86) is then solved, subject to boundary conditions on the pressure. To complete the time-step, these solutions are combined (using Eq. 85 ) to find the physical velocity field $\mathbf{u}^{K}$.

The original work of Yeung and Yu [19] describes this procedure in detail and gives expressions for the difference operator $\mathcal{Q}$. In that work and the present one, the solution of $\hat{\mathbf{u}}$ is accomplished by a spectral collocation method. The solution of the Poisson equation is also performed via a spectral collocation method [20]. The high efficiency and accuracy of the techniques demonstrated in these works stems from the cylindrical geometry and the decomposition of the solution into spectral modes.

\subsection{Matching techniques}

\subsubsection{Euler equation advancement of the matching boundary condition}

Most of the work that has been done regarding viscous-inviscid domain decomposition with regard to free-surface flows makes use of the Euler integral for inviscid flows to provide a mechanism to advance the solution and perform the matching. Campana [3] presents an example of this effort that is limited to two-dimensional flows in which the viscous flow lies above the inviscid flow and the matching interface does not intersect the free surface. That work adopts a Dirichlet to Neumann (DtN) approach of implementing a boundary-element solution to the inviscid-flow problem as a boundary condition on the viscous region. It is suspected that this technique will be unstable for the surface-piercing matching surface. A NtD version of the Euler-equation advancement technique which is stable is 
developed below for three-dimensional flows. This technique can be thought of as surrounding the viscous fluid by a circular surface that behaves as a wave-maker, moving in the same manner as the inviscid flow it replaces. This analogy is useful in understanding some of the limitations described below of this technique in the surface-piercing case.

In the Euler-equation advancement technique described by Campana [3], the Euler equation is integrated in time to provide a means to advance the velocity potential in the outer region at a new time-step, based upon the velocity and pressure field in the viscous region at the last time-step. This velocity potential is then used as a Dirichlet boundary condition for the solution of the potential flow in the inviscid region. This solution then provides a pressure and velocity boundary condition on the viscous flow at the new time-step. By discarding the quadratic terms in the Euler equation, this process can be diagrammed as:

DtN Scheme

$$
\begin{array}{rl|l}
\frac{\text { Viscous region }}{\mathbf{u}^{K-1}, P^{K-1}} & & \text { Inviscid region } \\
& \varphi^{K}=\varphi^{K-1}+\Delta t\left(P^{K-1}\right) \\
& \downarrow \text { Shell solution (DtN) } \\
\mathbf{u}^{K}, \partial P^{K} / \partial r= & \varphi_{r}^{K}
\end{array}
$$

Experience with this DtN use of the shell-function solution as a boundary condition suggests that this method may be unstable in the case here where the matching surface intersects the fluid-atmosphere interface. In fact, even in the submerged-interface case a very small time-step was required to achieve stability [3].

In order to avoid the DtN application of the shell-function solution, a modified approach is possible in which the normal derivative of the Euler equation is used to advance $\varphi_{r}$ in time, allowing the NtD version of the shellfunction solution to be used. The result is then a boundary condition for the viscous flow on the normal velocity at the matching surface, along with a Dirichlet condition for pressure. The information flow for this case can be diagrammed as:

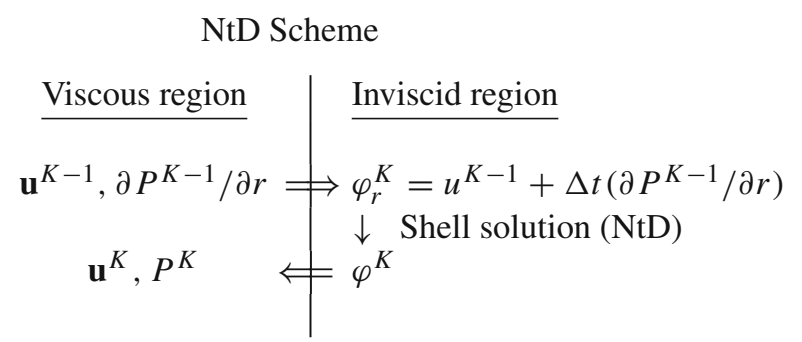

In order to achieve sufficient accuracy and stability, this procedure needs to be implemented as a predictorcorrector scheme. The predictor-step normal derivative of the outer potential is computed as a linear extrapolation from the viscous-flow quantities at the previous two time-steps:

$\varphi_{v}^{\bar{K}}\left(r_{o}, \theta, z\right)=2 u^{K-1}-u^{K-2}$.

This is used as a Neumann condition for the outer flow which provides a new outer potential $\varphi^{\bar{K}}$. For this predictor-step, the pressure on the shell surface at the new time-step is obtained from a backward-difference form of the linearized Euler integral:

$P^{\bar{K}}=\frac{\varphi^{\bar{K}}-\varphi^{K-1}}{\Delta t}$.

Application of this pressure as a boundary condition on the interior problem provides a better estimate for the radial velocity at the new time-step,

$\varphi_{v}^{K}=u^{K-1}+\frac{u^{\bar{K}}-u^{K-2}}{2}$, 
which gives the final pressure boundary condition for the interior region after (21) is used again:

$P^{K}=\frac{\varphi^{K}-\varphi^{K-1}}{\Delta t}$.

For the viscous inner flow, care must be exercised in the tangential velocities. While derivatives of the outer potential could also be used as tangential velocity boundary conditions for the inner flow, consistent results are achieved by implementing a free-slip velocity boundary condition on the shell surface. This approach is based on the physical idea that in this type of viscous-inviscid matching, the viscous flow is assumed to be bounded by a friction-less wall that moves in accordance with the outer inviscid flow. Unfortunately, this analogy has a limitation in that a viscous fluid bounded by a friction-less wall must, by definition, have zero slope at the intersection of the fluid-atmosphere interface and the friction-less wall. This is required to ensure a zero-stress condition at that point and a free-slip along the wall surface. Some success can be obtained with this matching approach. However, eventually the enforcement of such a zero-wave-slope condition at the matching surface causes the interior and exterior solutions to diverge sufficiently which creates an instability in the updating of the external radial velocity via the Euler equation. Besides, there is no reason a priori that the shell surface should be points of zero wave-slope. It is possible to implement a fix to this problem by relaxing the dynamic boundary conditions on the fluid-atmosphere intersection near the intersection contour of the fluid-atmosphere interface and the viscousinviscid interface. Instead of pursuing this further, a different advancement procedure of the solution utilizing a pressure-driven boundary condition is developed in the next section.

\subsubsection{Pressure-driven matching at shell surface}

An alternative technique is developed here that relies on implementing the boundary conditions for the viscousinviscid boundary. This technique advances the boundary conditions on the matching interface in a manner similar to the "pressure-driven" boundary condition implemented for the fluid-atmosphere free-surface conditions. The difficulties that appear in the advancement technique utilizing the Euler equation are avoided and excellent matching behavior is achieved.

In this matching technique, the coupling between the kinematics of the flow and the dynamic boundary conditions takes place through the solution of the inviscid outer-flow problem. Again, a predictor-corrector technique is implemented that can be performed in parallel with the predictor-corrector advancement of the fluid-atmosphere boundary-condition advancement. As before, the boundary-conditions on the auxiliary velocity field $\hat{u}$ are provided from the previous time-step:

$$
\left.\begin{array}{l}
\hat{u}=f_{1}\left(\Delta t, u^{K-1}, v^{K-1}, w^{K-1}, \nabla P^{K-1}\right) \\
\hat{v}=f_{2}\left(\Delta t, u^{K-1}, v^{K-1}, w^{K-1}, \nabla P^{K-1}\right) \\
\hat{w}=f_{3}\left(\Delta t, u^{K-1}, v^{K-1}, w^{K-1}, \nabla P^{K-1}\right)
\end{array}\right\} \quad \text { on } r=r_{o},
$$

Boundary conditions on the predicted pressure (denoted by the overbar on the superscript) is given by inserting quantities from the previous time-step in the dynamic boundary condition on the matching surface:

$$
P^{\bar{K}}=P_{o}^{K-1}+\frac{2}{\operatorname{Re}} \frac{\partial u^{K-1}}{\partial r} \text { at } r=r_{o} .
$$

Here, the pressure from the outer flow at the last time-step $\left(P_{o}^{K-1}\right)$ plays the role of the free-surface elevation in the fluid-atmosphere case. The $u^{\bar{K}}$ and $P^{\bar{K}}$ field values are then solved by using (84), (85), and (86). This solution is then adjusted in the same manner as in the fluid-atmosphere boundary-condition advancement. The velocities are forced to satisfy the dynamic boundary conditions on the interface surface:

$$
\left.\begin{array}{l}
\frac{\partial u^{\bar{K}}}{\partial r}=\frac{\operatorname{Re}}{2}\left(P^{\bar{K}}-P_{o}^{K-1}\right) \\
\frac{\partial w^{\bar{K}}}{\partial r}=-\frac{\partial u^{\bar{K}}}{\partial z} \\
r \frac{\partial}{\partial r}\left(\frac{v^{\bar{K}}}{r}\right)=-\frac{1}{r} \frac{\partial u^{\bar{K}}}{\partial \theta}
\end{array}\right\} \quad \text { at } r=r_{o} .
$$


At this point, instead of integrating the kinematic free-surface condition as done in the fluid-atmosphere case above, the shell-function solution of the outer flow provides a new outer pressure $\left(P_{o}^{\bar{K}}\right)$, based upon $u^{\bar{K}}$ as a boundary condition.

As in the fluid-atmosphere case, for the corrector step the auxiliary velocity $\hat{u}$ is not re-computed for the corrector step, and only the pressure field and actual velocity field are corrected. Boundary conditions on pressure for the corrector step are computed from the predicted solution,

$$
P^{K}=P_{o}^{\bar{K}}+\frac{2}{\operatorname{Re}} \frac{\partial u^{\bar{K}}}{\partial r} \text { at } r=r_{o}
$$

The pressure field and velocity field are then re-computed everywhere by solving (86) and (85) a second time. This corrected solution is again adjusted to ensure compliance with the dynamic boundary conditions,

$$
\left.\begin{array}{l}
\frac{\partial u^{K}}{\partial r}=\frac{\operatorname{Re}}{2}\left(P^{K}-P_{o}^{\bar{K}}\right) \\
\frac{\partial w^{K}}{\partial r}=-\frac{\partial u^{K}}{\partial z} \\
r \frac{\partial}{\partial r}\left(\frac{v^{K}}{r}\right)=-\frac{1}{r} \frac{\partial u^{K}}{\partial \theta}
\end{array}\right\} \quad \text { at } r=r_{o} .
$$

The outer shell-function solution is used again to compute the final outer pressure based upon the final radial velocities. This procedure is nearly the same as the advancement of the fluid-atmosphere boundary condition developed by $\mathrm{Yu}$ [21], but instead of using the kinematic boundary condition on wave elevation to drive the flow, the solution of the inviscid equations of motion in the outer region performs this role.

\subsection{Solution and results}

In previous works the inviscid outer solution has been effectively used as a radiation boundary condition for an interior viscous-flow solution, absorbing waves generated in the interior domain without reflection [6]. In this paper that idea has been extended to the case where waves propagate from the exterior flow into the interior region and diffract around a column located within the viscous solution region. The resulting wave-field that forms as a combination of the incident wave and the diffracted wave passes out of the viscous region and is absorbed by the shell without reflection.

When it is assumed that a potential-flow solution representing an appropriate incident wave is known, incident waves can be incorporated into the outer flow and matched to the inner viscous flow without interfering with the wave-absorbing characteristics of the shell-function outer boundary condition. It is critical that this incident-wave potential satisfies the same boundary and initial conditions assumed by the shell-function solution. As long as this is true, linear superposition of solutions in the outer region allows the incident-wave velocity potential to simply be added to the shell-function solution.

Figure 6 shows snapshots of the flow as the incident waves develop to a time-harmonic steady state. The diffracted waves here are less obvious than in the transient case of the inviscid flow. However, the presence of the diffracted wave is still visible as a distortion from a two-dimensional form of the wave-field. Figure 7 shows details of the viscous flow field in the plane $\theta=180$, where the incident waves are propagating into the viscous region from the left. Several snapshots are shown within one period of the incident waves. Figure 8 compares the resulting horizontal force on the cylinder with the inviscid-fluid computations. The pressure forces resulting from the viscous flow are similar to the inviscid results in the early part of the simulation but ultimately have a smaller magnitude in the steady-state response. A viscous force due to shear stress is also present but remains only a few percent of the pressure force since flow separation has not occurred.

Flow separation is an important feature of this type of flow, but is unlikely to occur when the linearized wave form is assumed. The small-wave-height linearization has the effect of guaranteeing that the convective terms on 

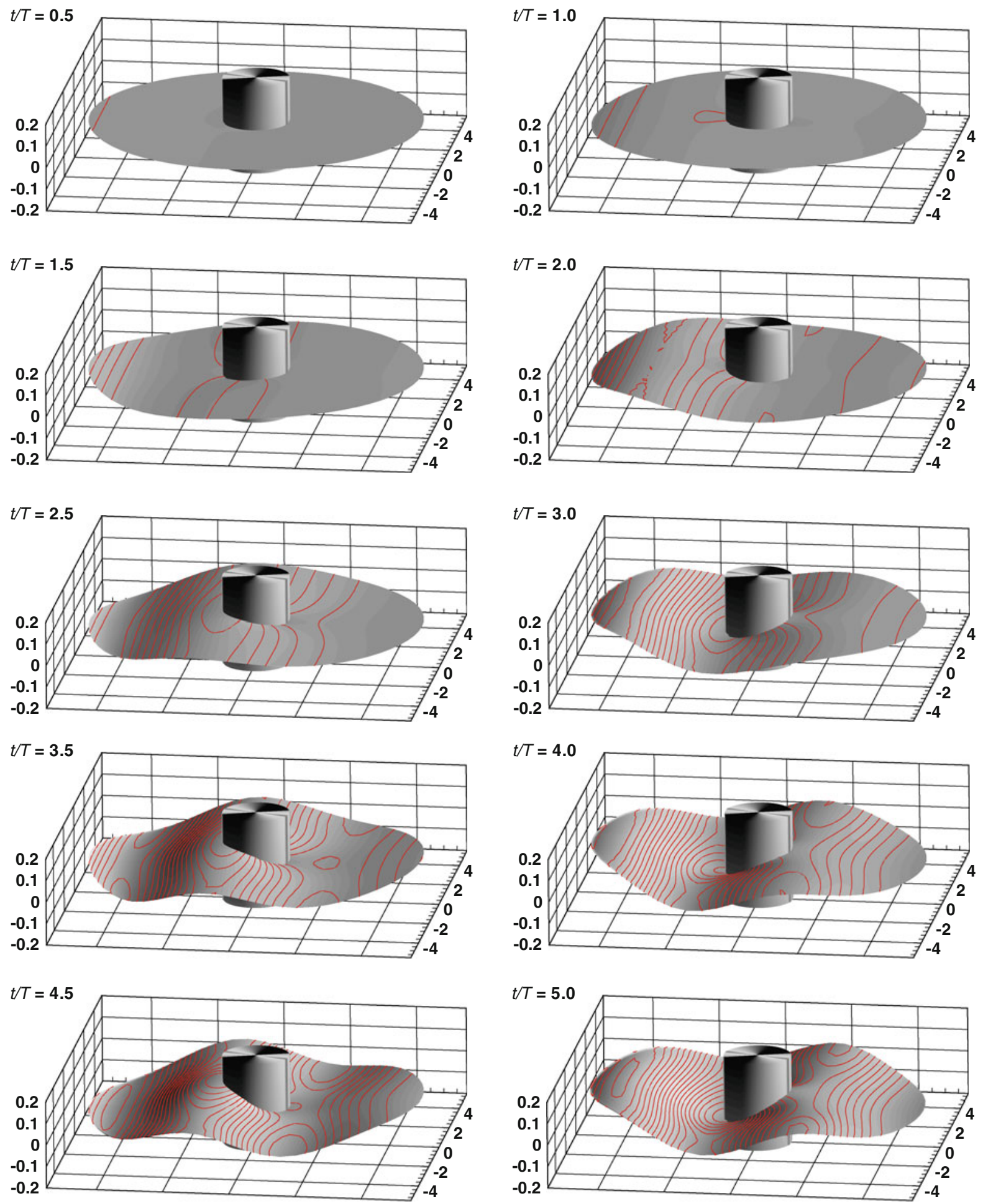

Fig. 6 Snapshots of the viscous interior region as incident waves propagate in from the outer region and diffract about the cylinder 

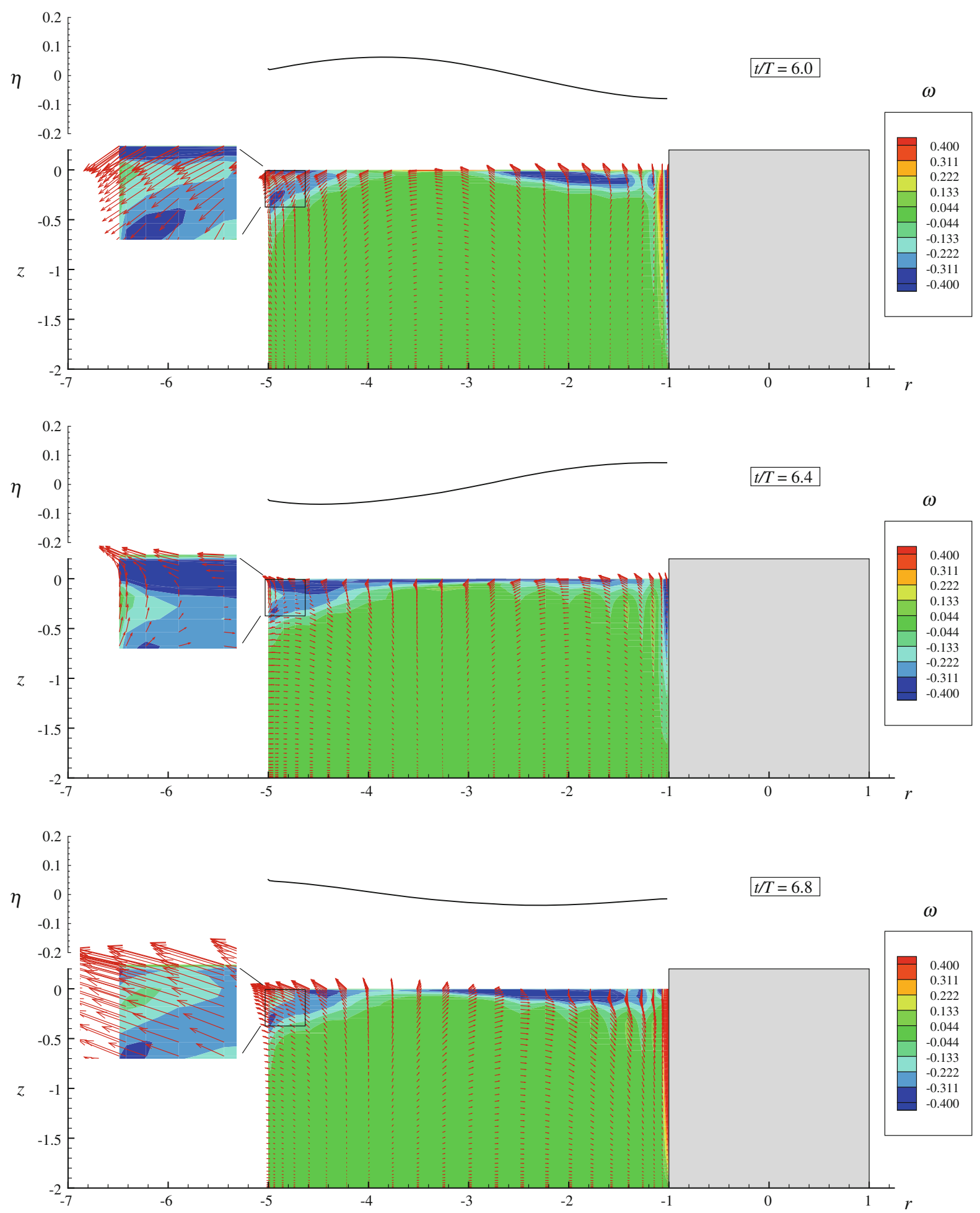

Fig. 7 Velocity vectors and vorticity contours "upstream" of cylinder as waves propagate into the viscous region from the left. Three snapshots are shown within one incident wave period $(T)$ after the flow has become time-harmonic 


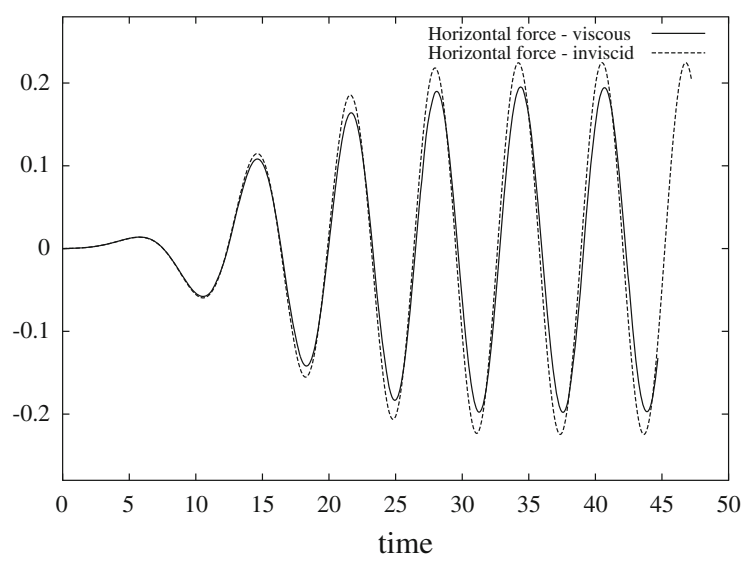

Fig. 8 Horizontal force on cylinder in an interior viscous region for incident waves generated by an oscillating pressure patch in the inviscid exterior region. Comparison is made to the fully inviscid case. Force and time are nondimensionalized by $\tilde{\rho} \tilde{g} \tilde{r}_{i}^{3}$ and $\sqrt{\tilde{r}_{i} / \tilde{g}}$, respectively

the left-hand side of the Navier-Stokes equations remain small relative to the inertial terms. Only in the case of a small cylinder diameter relative to the wave amplitude will the convective terms gain significance, resulting in flow separation behind the cylinder. An analogous way to see this is to consider the Keulegan-Carpenter number. For the oscillating flow due to the incoming waves, the $K C$ number may be defined as $2 \pi A / D$ where $A$ is the amplitude of the incoming wave and $D$ is the cylinder diameter. Sarpkaya [22] demonstrates that, for a simple oscillatory flow by a circular cylinder (no free surface), separation does not occur until the $K C$ number approaches 10 . For flow due to a linearized free-surface wave, this requires a cylinder diameter of approximately $30 \%$ of the wave amplitude, which is much smaller than the cylinder diameter used for the results presented in Fig. 6.

\section{Conclusions}

This paper presents a generalized solution of all possible inviscid, linear free-surface flows in a region extending outwards of a cylindrical surface. This generalized solution is used on a matching surface (the shell), which behaves like a non-axisymmetric cylindrical wave-maker coupled to the motion of the interior viscous-fluid region. In the procedure presented, the interior solution can match up exactly with the outer linear solution, time-step to time-step, as if the matching boundary is transparent. To implement this idea, appropriate flow variables (pressures and velocities acting on the interior region) need to be passed between the two solutions and in the proper order. This is a main contribution of this paper. Additionally, since the outer solution contains a spectrum of waves, its computations need to be rapid and efficiently. Otherwise, there would be no savings when compared to simply solving the equations of the interior flow in an awkwardly large domain. The introduction of a spectral solution form for the outer solution has facilitated solving the outer solution.

Since the exterior region is an inviscid, linearized surface-wave problem, with a cylindrical geometry, the generalized solution can be simplified by angular symmetry and quantified by a finite set of shell coefficients. The pre-computation and storage of these will subsequently provide a representation of the exterior wavy flow for any interior solutions. In fact, once the generalized solution is computed, stored, and retrieved, the computational burden for the exterior flow becomes only a small effort compared to solving the flow for the interior region.

Previous works [9] have shown this generalized exterior solution matched to an inviscid linear free-surface flow in the interior region. In that case, there is no discontinuity of field equations or boundary conditions across the matching boundary and a seamless matching is achieved. This paper extends this technique to the considerably more complex case of matching a viscous interior flow to an inviscid outer flow, with linear free-surface boundary conditions in both regions. As described in the paper, the methodology to achieve this relies on an iterative 
predictor-corrector scheme, which is tightly integrated into the fractional-step method of solving the Navier-Stokes equations.

An interesting, but so far unexplored, case is the matching of an interior flow region with nonlinear free-surface boundary conditions to the generalized solution of the linear exterior region flow. Obviously, as steep waves approach the matching boundary, the absorbtion would be imperfect and energy would reflect or cause other disturbances. However, for three-dimensional problems, steep waves will attenuate as they propagate outwards and the discontinuity between boundary conditions may become manageable. One could consider applying artificial damping to suppress only the portion of the outgoing wave not characterized by the linear solution in the exterior region. Such an approach warrants further study since exciting techniques of free-surface-flow simulation, such as smoothed particle hydrodynamics, are now enabled by the advent of powerful graphical processors [23,24], but the problem of absorbing outgoing wave energy in these methods will remain.

Acknowledgment R. W. Yeung acknowledges partial support for the preparation of this article under Office of Naval Research Grant No. N00014-09-1086 at UC Berkeley. J. A. Hamilton acknowledges support from the David and Lucille Packard foundation.

Open Access This article is distributed under the terms of the Creative Commons Attribution Noncommercial License which permits any noncommercial use, distribution, and reproduction in any medium, provided the original author(s) and source are credited.

\section{References}

1. Lai CH, Cuffe AM, Pericleous KA (1996) A domain decomposition algorithm for viscous/inviscid coupling. Adv Eng Softw 26:151-159

2. Drela M, Giles MB (1996) Viscous-inviscid analysis of transonic and low Reynolds number airfoils. AIAA J 25

3. Campana EF, Iafrati A (2001) Unsteady free surface waves by domain decomposition approach. In: Proceedings of the 16th international workshop on water waves and floating bodies, Kuju, Japan, April

4. Campana E, Di Mascio A, Esposito F, Lalli F (1995) Viscous-inviscid coupling in free surface ship flows. Int J Numer Methods Fluids 21:699-722

5. Guillerm PE, Alessandrini B (2003) 3D free-surface flow computation using a RANSE/Fourier-Kochin Coupling. Int J Numer Methods Fluids 43(3):301-318

6. Yeung RW, Hamilton JA (2002) A spectral-shell solution for viscous wave-body interaction. In: Proceedings of the 24th symposium on naval hydrodynamics, Fukuoka, Japan

7. Wehausen JV, Laitone EV (1960) Surface waves. In: Flugge S (ed) Handbuch der Physik, vol 9. Springer-Verlag, Berlin, pp 446-778

8. Yeung RW (1982) Transient heaving motions of floating cylinders. J Eng Math 16:97-119

9. Hamilton JA, Yeung RW (1997) Shell-function solution for 3-D nonlinear body-motion problems. Schiffstechnik, pp 62-70

10. Newman JN (1986) Distribution of sources and normal dipoles over a quadrilateral panel. J Eng Math 20:113-126

11. Coakley PS (1995) A high-order B-spline based panel method for unsteady, nonlinear, three-dimensional free-surface flows. PhD thesis, University of California at Berkeley

12. Beck RF, Liapis SJ (1987) Transient motions of floating bodies at zero forward speed. J Ship Res 31(3):164-176

13. Newman JN (1985) Algorithms for the free-surface Green function. J Eng Math 19:57-67

14. Press WH, Teukolsky SA, Vetterling WT, Flannery BP (1992) Numerical recipes in C. The art of scientific computing. Cambridge University Press. ISBN:0-521-43108-5

15. Finkelstein AB (1957) The initial value problems for transient water waves. Commun Pure Appl Math 10:511-522

16. Yeung RW, Yu X (2001) Three-dimensional free surface flow with viscosity—a spectral solution. In: Kashiwagi M (ed) Hydrodynamics in ships and ocean engineering. Research Institute of Applied Mechanics, Kyushu University, Japan, pp 87-114

17. Chorin AJ (1968) Numerical solution of incompressible flow problems. Stud Numer Anal 2:64-71

18. Yeung RW, Ananthakrishnan P (1992) Oscillation of a floating body in a viscous fluid. J Eng Math 26:211-230

19. Yeung RW, Yu X (1994) Three-dimensional flow around a surface-piercing body. In: Proceedings of the 20th symposium on naval hydrodynamics, Santa Barbara, CA

20. Yu X, Yeung RW (1995) Interaction of transient waves with a circular surface-piercing body. J Fluids Eng 117:382-388

21. Yu X (1996) Free-surface flow around a vertical strut in a real fluid. PhD thesis, University of California at Berkeley

22. Sarpkaya T (1986) Force on a circular cylinder in viscous oscillatory flow at low Keulegan-Carpenter numbers. J Fluid Mech 165:61-71

23. Harada T, Koshizuka S, Kawaguchi Y (2007) Smoothed particle hydrodynamics on GPUs. In: Proceedings of the 25th computer graphics international conference, Petropolis, Brazil, pp 63-70

24. Herault A, Bilotta G, Dalrymple R (2010) SPH on GPU with CUDA. J Hydraul Res 48:74-79 